\title{
A BIDIAGONALIZATION-REGULARIZATION PROCEDURE FOR LARGE SCALE DISCRETIZATIONS OF ILL-POSED PROBLEMS*
}

\author{
DIANNE P. O’LEARY† AND JOHN A. SIMMONS $\ddagger$
}

\begin{abstract}
In this paper, we consider ill-posed problems which discretize to linear least squares problems with matrices $K$ of high dimensions. The algorithm proposed uses $K$ only as an operator and does not need to explicitly store or modify it. A method related to one of Lanczos is used to project the problem onto a subspace for which $K$ is bidiagonal. It is then an easy matter to solve the projected problem by standard regularization techniques. These ideas are illustrated with some integral equations of the first kind with convolution kernels, and sample numerical results are given.
\end{abstract}

Key words. ill-posed problems, Lanczos algorithm, regularization, first kind integral equation

1. Introduction. In this paper we discuss techniques applicable to the solution of those ill-posed problems which, upon discretization, give rise to large linear systems of equations. In particular, our examples are drawn from integral equations of the first kind,

$$
\int_{a}^{b} k(s, t) f(t) d t=g(s)
$$

or

$$
\min _{f}\left\|\int_{a}^{b} k(s, t) f(t) d t-g(s)\right\|^{2}
$$

which discretize to the linear system

$$
\begin{aligned}
K f=g, & K: m \times n, \quad m \geqq n, \\
& f: n \times 1, \\
& g: m \times 1
\end{aligned}
$$

or to the minimization problem

$$
\min _{f}\|K f-g\|_{2}^{2} .
$$

Such continuous problems are characterized by the fact that small changes in the function $g$ can cause large changes in $f$. This is reflected in the discrete problem by ill-conditioning in the matrix $K$. Since such perturbations can be due to unavoidable noise in measurements of $g$ or to roundoff errors in the calculation, algorithms for numerical solution of the discretized problem must be designed to minimize the effects of these perturbations.

Various techniques for solving linear ill-posed problems are discussed in a good survey paper by Björck and Eldén [3]. We do not attempt an exhaustive review of

* Received by the editors September 29, 1980.

† Mathematical Analysis Division, National Bureau of Standards, Washington, DC 20025, and Computer Science Department and Institute for Physical Science and Technology, University of Maryland, College Park, Maryland 20742.

$\ddagger$ Metallurgy Division, National Bureau of Standards, Washington, DC 20025 . 
these techniques here, but note that two of the most popular methods are based on the following techniques:

(1) Regularization. We use this term in a broad sense [3] to describe methods which replace the original operator by a related one which diminishes the effects of errors in the data. For example, the function to be minimized might be replaced by

$$
\min _{f}\|K f-g\|_{2}^{2}+\gamma\|f\|_{L}^{2}
$$

where $\|f\|_{L}^{2}=f^{*} L^{*} L f$ for some full rank matrix $L, \gamma$ is a positive scalar parameter, and the superscript $*$ denotes complex conjugate transpose. This is equivalent to solving the system of equations

$$
\left(K^{*} K+\gamma L^{*} L\right) f=K^{*} g .
$$

Thus the operator $K^{*} K$ of the normal equations for the original problem has been replaced by an operator $K^{*} K+\gamma L^{*} L$. The choices of $\gamma$ and $L$, guided by the physical characteristics of the problem and of the noise, give a problem for which the operator is better conditioned but which has a solution close, in some sense, to that of the original problem.

(2) Projection. The approximate solution $f$ is constrained to lie in a specified subspace given by the columns of a matrix $V$. In this case we have a modified problem,

$$
\min _{h}\|K V h-g\|_{2}^{2}
$$

or

$$
V^{*} K^{*} K V h=V^{*} K^{*} g, \quad f=V h .
$$

The new operator, $V^{*} K^{*} K V$, is $K^{*} K$ restricted to a subspace upon which it is better conditioned.

The technique we consider is a projection-regularization method. In the first step, the problem is projected onto a subspace defined by a bidiagonalization algorithm. The restricted operator is typically still ill-conditioned. In the second step a regularization is applied on the subspace. The reason for this approach is that regularization of the restricted problem can be less expensive and, if the subspace is chosen properly, the final results are not significantly degraded. The algorithm and its properties are presented in $\S 2$. Potential applications of the algorithm include:

(i) Problems for which $n$ multiplications by the operator $K$ are significantly less expensive than factorization of the matrix.

(ii) Problems for which storage does not permit regularization of the original problem.

Thus, problems for which $K$ is sparse or $K$ is structured so that its storage and matrix-vector multiplication time are both less than $O(m n)$ are possible candidates for this algorithm. Examples of such problems and sample computational results are given in $\S 4$.

2. The bidiagonalization-regularization algorithm. The algorithm proceeds in two steps. First the problem is reduced to one on a subspace which is computationally much more economical. Then standard techniques of regularization can be used on the reduced problem. The two steps are defined in $\$ \S 2.1$ and 2.2, and properties of the algorithm are discussed in $\S 2.3$. A different algorithm based on bidiagonalization has been proposed independently in [2]. 
2.1 The Lanczos bidiagonalization. The subspace chosen over which to solve the problem is that generated by the "Lanczos" algorithm for bidiagonalization. This algorithm was investigated by Paige [20], named and described in block form by Golub, Luk and Overton [11] and used in a different context by Moler and Stewart [19]. It is a specific computational implementation of the bidiagonalization procedure of Golub and Kahan [10], proposed as the first step of computing the singular value decomposition of a matrix. It is related to ideas of Lanczos [16] and [17].

The Lanczos bidiagonalization algorithm takes an $m \times n$ matrix $K$ and factors it as

$$
\begin{array}{ll}
U^{*} K Q=B, & U: m \times m, \\
& B: m \times n, \\
& Q: n \times n
\end{array}
$$

or

$$
K=U B Q^{*}
$$

where $U$ and $Q$ are orthonormal,

$$
U^{*} U=I, \quad Q^{*} Q=I,
$$

and $B$ is bidiagonal,

$$
B=\left[\begin{array}{lllllll}
\alpha_{1} & \beta_{1} & & & & \\
& \alpha_{2} & \beta_{2} & & & \\
& & \cdot & \cdot & \cdot & \\
& & & \cdot & \cdot & \beta_{n-1} \\
& & & & & \alpha_{n} \\
& & & & & \\
\hline & & & & 0 &
\end{array}\right] .
$$

This is done using very elementary manipulations: we need to form the product of $K$ and $K^{*}$ with various vectors and to take linear combinations and inner products of vectors. Furthermore, rather than carrying out the full algorithm, it can be terminated early to give a factorization of $K$ as an operator over a subspace:

$$
\begin{array}{ll}
U_{k}^{*} K Q_{k}=B_{k}, & U_{k}: m \times k, \\
& Q_{k}: n \times k, \\
& B_{k}: k \times k,
\end{array}
$$

where

$$
\begin{gathered}
B_{k}=\left[\begin{array}{ccccccc}
\alpha_{1} & \beta_{1} & & & & \\
& \alpha_{2} & \beta_{2} & & & \\
& & \cdot & \cdot & \cdot & \\
& & \cdot & \cdot & \beta_{k-1} \\
& & & & \alpha_{k}
\end{array}\right], \\
U_{k}^{*} U_{k}=I, \quad Q_{k}^{*} Q_{k}=I .
\end{gathered}
$$


The matrix $B$ has the same singular values as $K$, and the singular values of $B_{k}$ can be shown to be close to certain of $K$ 's, typically its largest and smallest [11].

The algorithm can be derived from the relations $K Q=U B$ and $U^{*} K=B Q^{*}$. It proceeds as follows [11].

Given $K: m \times n$ and $z_{1}: n \times 1$ an arbitrary nonzero vector, set

$$
q=\frac{z_{1}}{\left\|z_{1}\right\|}, \quad y_{1}=K q_{1}, \quad \alpha_{1}=\left\|y_{1}\right\|, \quad u_{1}=\frac{y_{1}}{\alpha_{1}} .
$$

For $i=1,2, \cdots, k-1$,

$$
\begin{aligned}
& z_{i+1}=K^{*} u_{i}-\alpha_{i} q_{i}, \quad \beta_{i}=\left\|z_{i+1}\right\|, \\
& q_{i+1}=\frac{z_{i+1}}{\beta_{i}}, \\
& y_{i+1}=K q_{i+1}-\beta_{i} u_{i}, \quad \alpha_{i+1}=\left\|y_{i+1}\right\|, \\
& u_{i+1}=\frac{y_{i+1}}{\alpha_{i+1}} .
\end{aligned}
$$

The vector $q_{i}\left(u_{i}\right)$ is the $i$ th column of the matrix $Q(U)$.

Operations counts and properties of the algorithm will be discussed in $\S 2.3$.

2.2 Regularization of the bidiagonal problem. Our original discretized problem was to solve

$$
\min _{f}\|K f-g\|_{2}^{2}
$$

If we decide to consider only those vectors $f$ contained in the subspace spanned by the columns of $Q_{k}$, i.e.,

$$
f=Q_{k} h \text { for some } h: k \times 1 \text {, }
$$

and try to minimize the residual only in the directions spanned by the columns of $U_{k}$, we have the reduced problem

$$
\min _{h}\left\|U_{k}^{*}\left(K Q_{k} h-g\right)\right\|_{2}^{2}=\min _{h}\left\|B_{k} h-U_{k}^{*} g\right\|_{2}^{2}
$$

This problem is typically still highly ill-conditioned, because while the matrix $B_{k}$ usually contains very good approximations to the large singular values of $K[11]$, it also has singular values which are rough approximations to the small ones. Thus forming and solving the normal equations

$$
B_{k}^{*} B_{k} h=B_{k}^{*} U_{k}^{*} g
$$

will probably not give an acceptable solution. On the other hand, we have reduced our problem to one of smaller dimension having a matrix that is bidiagonal. Thus, any of the standard regularization or projection procedures [3] will be economical. For example, we can use a truncated singular value decomposition, (SVD) expressing

$$
\begin{gathered}
B_{k}=V \Sigma W^{*}, \quad W^{*} W=I, \quad V^{*} V=I, \\
\Sigma=\operatorname{diag}\left(\sigma_{1}, \sigma_{2}, \cdots, \sigma_{k}\right), \quad \sigma_{1} \geqq \sigma_{2} \geqq \cdots \geqq \sigma_{k} \geqq 0,
\end{gathered}
$$


defining a truncated pseudo-inverse for $\Sigma$,

$$
\Sigma_{r}^{+}=\operatorname{diag}\left(\frac{1}{\sigma_{1}}, \cdots, \frac{1}{\sigma_{r}}, 0, \cdots, 0\right),
$$

and then setting

$$
h=W \Sigma_{r}^{+} V^{*} U_{k}^{*} g=\sum_{i=1}^{r} \frac{v_{i}^{*} U_{k}^{*} g}{\sigma_{i}} w_{i}
$$

where $w_{i}\left(v_{i}\right)$ is the $i$ th column of $W(V), \Sigma_{r}=\operatorname{diag}\left(\sigma_{1}, \cdots, \sigma_{r}, 0, \cdots, 0\right), \sigma_{r}>0$, and the superscript "+" denotes the pseudo-inverse. This method might be considered either a regularization method or a projection method.

As another alternative we could take a damped SVD approach, substituting $\alpha_{i} / \sigma_{i}$ for $1 / \sigma_{i}$ in the above expression for $h$, where the $\alpha_{i}$ form a sequence of decreasing positive numbers. The damping factors might be determined using generalized cross validation techniques [9], [28].

These SVD approaches are $O\left(k^{3}\right)$ processes. Even more efficient methods have been suggested by Eldén [6] [7] and Gander [8], basing the regularized solution directly on the bidiagonal form and producing a solution in $O\left(k^{2}\right)$ operations.

2.3 Properties and extensions of the algorithm. For definiteness in the discussion, we assume in this section that a truncated singular value regularization is performed.

(A) The operations count for the Lanczos bidiagonalization is $3(n+m) k$ multiplications plus $2(n+m) k$ additions plus $k$ matrix-vector multiplications involving $K$ and $k-1$ involving $K^{*}$. The SVD, as mentioned above, is $O\left(k^{3}\right)$, and the final solution is calculated in $(n+m) k$ additions and multiplications. Thus if $k$ is small compared with $n$, significant savings can be realized with respect to the cost of the full singular value decomposition algorithm, an $O\left(n^{2} m\right)$ process.

(B) The Lanczos bidiagonalization can easily be implemented with $2 n+2 m+3 k$ storage locations in fast store $\left(q, K^{*} u, u, K q, \alpha, \beta, U^{*} g\right)$, with the vectors $q$ saved in auxiliary storage as they are computed. For the singular value decomposition, $2 k^{2}+k$ locations are required for $V, W$ and $\Sigma$. To compute the solution, these three matrices are used to form $h$, and then $f$ is formed in $O(n k)$ operations with access to the vectors $q$. Thus, for efficient implementation, the storage requirement is that required for $K$ plus max $\left(2 n+2 m+3 k, 2 k^{2}+2 k\right)$ in main storage, plus $n k$ in auxiliary to be written and read once sequentially. (By rearranging the algorithm slightly the storage of $V$ could be avoided.)

(C) If several problems are to be solved involving the same operator $K$, it can be more economical to also save the $u$ vectors. In this way, the Lanczos iteration and the singular value decomposition need to be performed only once.

(D) If there is not enough storage available to store the $q$ sequence, the $q$ vectors can be regenerated by running the Lanczos algorithm again using the same starting vector.

(E) For very ill-conditioned problems, reorthogonalization of the $q$ and $u$ vectors may be necessary to preserve computational stability [22], [5], [24]; although in theory $q_{i}^{*} q_{j}=u_{i}^{*} u_{j}=0, i \neq j$, in practice this may be far from being satisfied. In this case it may be necessary to perform modified Gram-Schmidt orthogonalization on the sequences. As each $z_{i}$ is calculated, we would perform the iteration

$$
\text { For } j=i-1, i-2, \cdots, 1, \quad z_{i} \leftarrow z_{i}-\left(z_{i}^{*} q_{j}\right) q_{j} \text {, }
$$


before normalizing $z_{i}$. A similar process would be performed on the $y$ sequence. This reorthogonalization can be done in a selective manner, depending on the magnitude of the inner product of the $i$ th and $j$ th vectors, or by running the loop from $i-1$ to $i-s$ for some $s<i$. In the case of full reorthogonalization the added cost is $(n+m) k^{2}$.

(F) There are two arbitrary parameters in the method: the number of Lanczos steps $(k)$ and the number of retained singular values $(r)$. The choice of these is problem dependent, and needs further investigation. Limited computational experience is summarized in $\S 4$.

(G) For certain singular value distributions, it may be advantageous to use the block Lanczos algorithm of Palmer [23] or Golub, Luk and Overton [11]. This can improve the convergence by reducing the number of vectors $q$ and $u$ necessary for an acceptable solution. The number of accesses to $K$ and $K^{*}$ is reduced by forming their products with several vectors at once; thus the method is also useful, regardless of the singular value distribution, if $K$ is stored in secondary memory and if there is room for one or more extra pairs of vectors of dimension $n$ and $m$ in main memory.

(H) "Preconditioning" techniques can be incorporated either to accelerate convergence or to change the character of the approximate solution. These two uses of preconditioning are described below.

(i) The problem

$$
\min _{f}\|K f-g\|_{2}^{2}
$$

is equivalent to

$$
\min _{\hat{f}}\|K N \hat{f}-g\|_{2}^{2}
$$

where $N$ is any nonsingular $n \times n$ matrix and $f=N \hat{f}$. If the singular value spectrum of $K N$ has better clustering of the singular values than does $K$, the number of Lanczos steps necessary can be significantly reduced. The price paid is an extra matrix-vector multiplication by $N$ and by $N^{*}$ at each iteration. This idea has been exploited previously in the solution of linear systems (e.g., [1], [4]) and certain least squares problems [26].

(ii) A common technique in many of these problems is "filtering" [13]. In this case $K$ and $g$ are both preconditioned by some operator $M$, changing the problem to

$$
\min _{\tilde{f}}\|M K \tilde{f}-M g\|_{2}^{2}
$$

Again the convergence rate is improved if the spectrum of $M K$ has good clustering properties. The regularized solution $\tilde{f}$ will be different from the $f$ above. The operator $M$ should be chosen to filter out undesirable components of $K$ and $g$, for example, highly oscillatory modes which are due to random errors in the measurements.

3. A time series deconvolution problem. We consider a special class of problems for which the algorithm in the previous section is applicable. In the case of interest, the integral equation

$$
\int_{a}^{b} k(s, t) f(t) d t=g(s)
$$

has a kernel of convolution type

$$
k(s, t)=k(s-t)
$$

and $b=s$. 
Upon discretization, our problem becomes $K f=g$, where

$$
K=\left[\begin{array}{lllll}
a_{0} & & & & \\
a_{1} & a_{0} & & & \\
\vdots & \vdots & & & \\
a_{n-1} & a_{n-2} & \ldots & a_{0}
\end{array}\right]
$$

Thus $K$ is lower triangular and Toeplitz. The time series involved are "causal," meaning that $k(t), f(t)$ and $g(t)$ are zero for negative values of their arguments. If $k$ is continuous, then $a_{j}$ is small for small values of $j$, and these small numbers near the main diagonal of $K$ cause ill-conditioning and prevent us from solving the system accurately by a simple forward substitution algorithm or by the faster algorithms for solving Toeplitz systems (e.g., [12]).

To understand the nature of this ill-conditioning, it is convenient to study a permuted version of the system, formed by reordering the equations from last to first:

$$
\left[\begin{array}{cccc}
a_{n-1} & a_{n-2} & \cdots & a_{0} \\
\vdots & \cdot & & \\
a_{0} & & &
\end{array}\right]\left[\begin{array}{c}
f_{0} \\
\vdots \\
f_{n-1}
\end{array}\right]=\left[\begin{array}{c}
g_{n-1} \\
\vdots \\
g_{0}
\end{array}\right] .
$$

We denote this reordered system by $K^{\#} f=g^{\#}$ and note that $K=P K^{\#}$, where $P$ is the permutation matrix formed by writing the rows of the identity matrix from last to first. $K^{\#}$ is a Hankel matrix and is indefinite.

Now $K$ is a defective matrix; it has $n$ eigenvalues equal to $a_{0}$ but only one eigenvector, the last unit vector $e_{n}=[0, \cdots, 0,1]^{T}$. But $K^{\#}$ is symmetric and thus has a full set of orthonormal real eigenvectors $w_{i}$ with eigenvalues $\sigma_{i}$ :

$$
K^{\#} w_{i}=\sigma_{i} w_{i}, \quad i=1,2, \cdots, n
$$

or

$$
K^{\#}=W \Sigma W^{T},
$$

where

$$
\begin{gathered}
W=\left[w_{1}, \cdots, w_{n}\right], \quad W^{T} W=I, \\
\Sigma=\left[\begin{array}{ccc}
\sigma_{1} & & \\
& \cdot & \\
& & \cdot \\
& & \sigma_{n}
\end{array}\right], \\
\left|\sigma_{1}\right| \geqq\left|\sigma_{2}\right| \geqq \cdots \geqq\left|\sigma_{n}\right| \geqq 0 .
\end{gathered}
$$

Thus $K=P K^{\#}=(P W) \Sigma W^{T}$ and this, aside from the signs of the $\sigma_{i}$ and those of the columns of $P W$, is the singular value decomposition of $K$. Therefore, we have a representation of $K$ as a sum of $n$ rank one matrices:

$$
K=\sum_{i=1}^{n} \sigma_{i} w_{i}^{\#} w_{i}^{T}, \quad w_{i}^{\#}=P w_{i}
$$

If $K$ is nonsingular $\left(a_{0} \neq 0\right)$, its inverse is

$$
K^{-1}=\sum_{i=1}^{n} \frac{1}{\sigma_{i}} w_{i}^{\#} w_{i}^{T} .
$$


If $K$ is singular $\left(a_{0}=0\right)$, we have the pseudo-inverse

$$
K^{+}=\sum_{i=1}^{r} \frac{1}{\sigma_{i}} w_{i}^{*} w_{i}^{T}
$$

where $\left|\sigma_{r}\right|>0, \sigma_{r+1}=\cdots=\sigma_{n}=0$.

From the decomposition above we can isolate the source of trouble in solving the linear system $K^{\#} f=g^{\#}$. (A similar analysis could be performed for the continuous problem.) Suppose that $a_{0}, \cdots, a_{s-1}$ are zero. Then

$$
K^{\#}=\left[\begin{array}{cc}
A & 0 \\
0 & 0
\end{array}\right]
$$

This matrix has $n-s$ eigenvalues equal to those of $A$, with eigenvectors of the form

$$
\left[\begin{array}{c}
\bar{w}_{i} \\
0
\end{array}\right], \quad \bar{w}_{i}:(n-s) \times 1
$$

and $s$ zero eigenvalues equal to those of $A$, with eigenvectors

$$
\left[\begin{array}{c}
0 \\
e_{i}
\end{array}\right]
$$

where the $e_{i}$ are $s \times 1$ unit vectors. If $a_{0}, \cdots, a_{s-1}$ are small rather than zero, the eigenstructure will be similar, but all of the zeros will be perturbed slightly [29]. The unregularized solution to the problem is given by

$$
f=\sum_{i=1}^{n} \frac{1}{\sigma_{i}} w_{i}^{T} g^{\#} w_{i}^{\#} .
$$

Thus there will be large contributions to the solution from the small singular values if $g^{\#}$ has any components in a direction $\left[0, e_{i}\right]^{T}, i=1, \cdots, s$. This means that noise in the first part of the vector $g$ will be magnified by large factors and added to the last part of the solution vector $f$.

In some of these problems it was observed that the Fourier vectors are approximate eigenvectors for $K^{\#}$. This can be explained by considering the problem in the frequency domain rather than the time domain. Let the matrix $F$ be defined by

$$
f_{j l}=\frac{1}{\sqrt{n}} e^{2 \pi i j l / n}, \quad l, j=0,1, \cdots, n-1, \quad i=\sqrt{-1}
$$

Then $F^{*} F=I$. Suppose the sequence $\left(a_{n-1}, \cdots, a_{0}\right)$ is band-limited; i.e.,

$$
F^{*}\left[\begin{array}{c}
a_{n-1} \\
\vdots \\
a_{0}
\end{array}\right]=\sum_{j \in J} \delta_{j} e_{j},
$$

where $J$ is an index set of cardinality $m$, small compared with $n$. Then if the norm of the vector $\left[a_{m-1}, \cdots, a_{0}\right]$ is small compared with the norm of the full vector $\left[a_{n-1}, \cdots, a_{0}\right]$, the columns of $F$ corresponding to indices in $J$ are an approximate invariant subspace of $K$; i.e., there is a subset of eigenvectors of $K$ whose span is approximately that of those columns. To see this we form

$$
F^{*} K^{\#} F=\left[\begin{array}{ll}
C_{1} & C_{2} \\
C_{3} & C_{4}
\end{array}\right], \quad C_{1}: m \times m
$$


(where $F$ is reordered if necessary to put the columns with indices $j \in J$ first) and show that $\left\|C_{3}\right\|$ is small. Then, by [27, Thm. 4.11], the desired result follows. Now

$$
F^{*} K^{\#}=\left[\delta, \delta+\left[\begin{array}{c}
\nu_{1} \\
\mu_{1}
\end{array}\right], \cdots, \delta+\left[\begin{array}{c}
\nu_{n-1} \\
\mu_{n-1}
\end{array}\right]\right]
$$

where $\delta=\left(\delta_{1}, \cdots, \delta_{m}, 0, \cdots, 0\right)^{T}$ and the vectors $\nu_{i}$ are $m \times 1$. Now, the norms of the first few vectors $\mu_{i}$ are small because the first few columns of $K^{\#}$ differ from its first column by small perturbation terms. The norms of later vectors $\mu_{i}$ are small because the norm of $\delta^{T}+\left[\nu_{i}^{T}, \mu_{i}^{T}\right]$ is equal to the norm of the $i$ th column of $K$, which is small compared with the norm of the first column. Thus $F^{*} K^{\#}$ has small entries in all rows not indexed by $J$, and therefore $F^{*} K^{\#} F$ is also small in those rows.

This Fourier property of the eigenvectors means that a truncated singular value decomposition often gives results indistinguishable from filtering.

In using the algorithm of $\S 2$, we can take advantage of three simplifications:

(1) Since $K^{\#}$ is symmetric, we can use the Lanczos tridiagonalization procedure [17], [21] rather than bidiagonalization. This requires approximately half of the work and storage, since the $u$ sequence is redundant, and only one matrix multiplication is needed per step. The resulting algorithm is:

Given $K^{\#}$ and $z_{1}: n \times 1$, an arbitrary nonzero vector, set

$$
q_{1}=\frac{z_{1}}{\left\|z_{1}\right\|}, \quad \Psi_{1}=1
$$

For $i=1,2, \cdots, k-1$

$$
\begin{aligned}
& z_{i+1}=K^{\#} q_{i}-\theta_{i} q_{i}-\Psi_{i} q_{i-1}, \\
& \theta_{i}=q_{i}^{T} K^{\#} q_{i}, \\
& \Psi_{i+1}=\left\|z_{i+1}\right\|, \\
& q_{i+1}=\frac{z_{i+1}}{\Psi_{i+1}} .
\end{aligned}
$$

The algorithm performs better numerically if $\theta_{i}$ is calculated as $q_{i}^{T}\left(K^{\#} q_{i}-\Psi_{i} q_{i-1}\right)$.

(2) Since the resulting $B$ is symmetric tridiagonal rather than bidiagonal, we can compute its eigendecomposition rather than its singular value decomposition. This cuts the storage required from $2 k^{2}+k$ to $k^{2}+k$, and also involves less computation.

(3) There exist fast algorithms for forming the product of $K^{\#}$ with an arbitrary vector. This arises from the relation of $K$ to a circulant matrix. Let

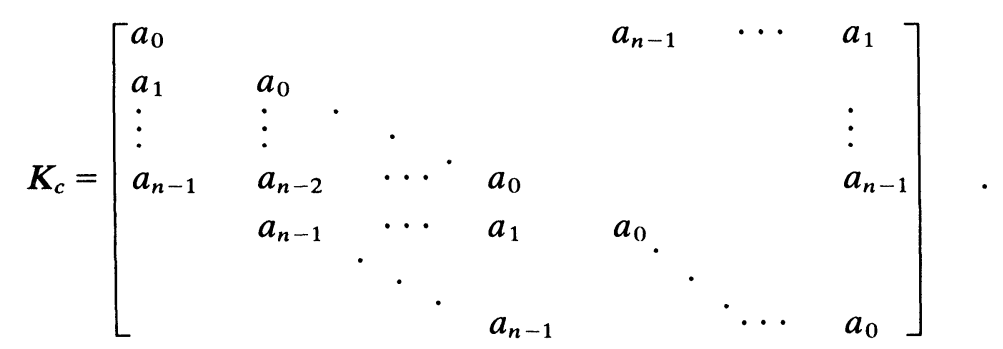

Then $K_{c}$ is a square circulant matrix of dimension $p \geqq 2 n-1$. The eigenvectors of any circulant matrix are the Fourier vectors, the columns of the $p \times p$ matrix $F$ defined 
above. The eigenvalues are given by the components of the Fourier transform of its first column:

$$
F\left[\begin{array}{l}
\alpha_{0} \\
\vdots \\
a_{n-1} \\
0 \\
\vdots \\
0
\end{array}\right]=\left[\begin{array}{l}
\lambda_{1} \\
\cdot \\
\cdot \\
\cdot \\
\lambda_{p}
\end{array}\right]
$$

Thus, $K_{c}=F \Lambda F^{*}$ where $\Lambda=\operatorname{diag}\left(\lambda_{1}, \cdots, \lambda_{p}\right)$. Note that to form the product of $K$ with an arbitrary vector $x$, we can form the product of $K_{c}$ with the vector $x$ padded by zeros, and take the first $n$ components of the result. To do this, the vector

$$
z=K_{c}\left[\begin{array}{l}
x \\
0
\end{array}\right]=F \Lambda F^{*}\left[\begin{array}{l}
x \\
0
\end{array}\right]
$$

is calculated in three steps:

(a) $\hat{x}=F^{*}\left[\begin{array}{l}x \\ 0\end{array}\right]$

(b) $y_{i}=\lambda_{i} \hat{x}_{i}, i=1,2, \cdots, p$,

(c) $z=F y$.

Then $K x$ is given by the first $n$ components of $z$. Step (b) costs $p$ operations, while (a) and (c) are Fourier transforms of $p$-vectors and cost $O\left(p \log _{2} p\right)$, for example, if $p$ is chosen to be a power of 2 .

4. Numerical examples. Two sample problems were chosen in order to demonstrate the algorithms of $\S 2$. The experiments were run in single precision on a Univac 1108 computer. In each case the initial Lanczos vector $z_{1}$ was taken to be $g$. Machine storage limited the number of Lanczos vectors for the largest problem to be less than or equal to 40 , so $k=25$ and 40 were taken as representative values. The number of singular values to be dropped was guided by the uncertainty in the data, but further investigation would be needed to make the procedure automatic.

Example 1. The following integral equation has been studied, for example, in [18], [25]:

$$
\begin{aligned}
& \int_{-6}^{6} k(s, t) f(t) d t=g(s), \quad|s| \leqq 6, \\
& k(s, t)= \begin{cases}1+\cos \frac{(t-s) \pi}{3}, & |t-s| \leqq 3, \\
0, & \text { otherwise, }\end{cases} \\
& g(s)= \begin{cases}(6-|s|)\left(1+\frac{1}{2} \cos \frac{s \pi}{3}\right)+\frac{9}{2 \pi} \sin \frac{|s| \pi}{3}, & |s| \leqq 6, \\
0, & \text { otherwise, }\end{cases}
\end{aligned}
$$

Solution: $f(s)=k(s, 0)$.

The right-hand side $g$ and the solution $f$ are plotted in Fig. 1. The integral equation was discretized using the trapezoidal rule with steplength $12 /(n-1)$. This yields a 


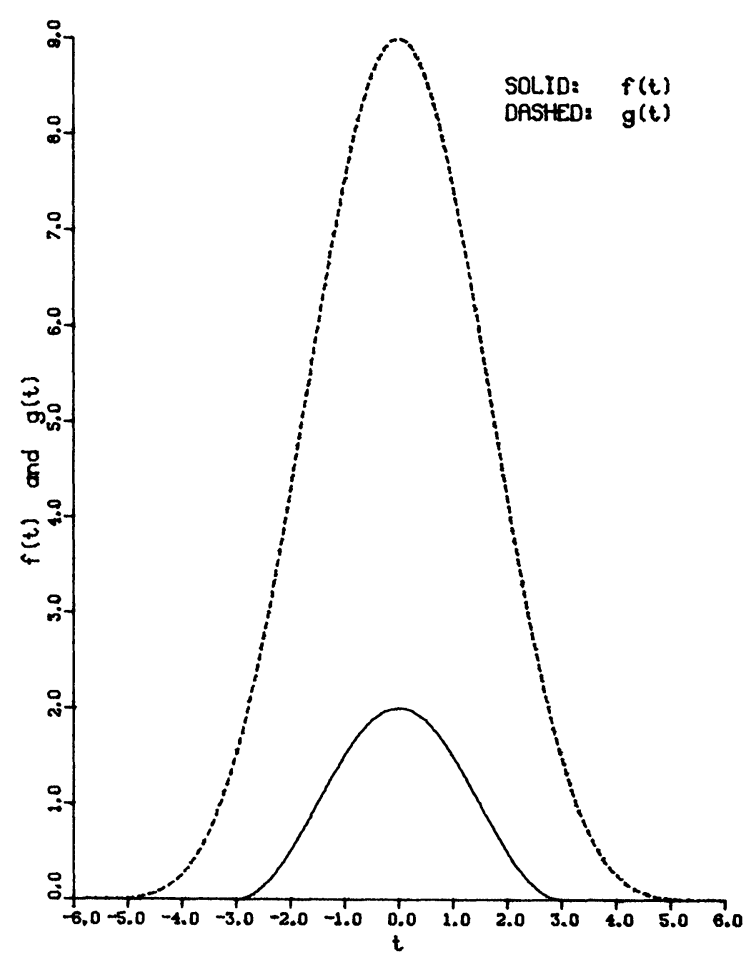

FIG. 1. Example 1, functions $f$ and $g$.

symmetric banded Toeplitz matrix if the first and last unknowns are replaced by their values divided by 2 ; see [18] for details. The discretization of $f$ does not satisfy the linear system exactly, but this discretization was taken as the "true" solution to the problem. The Lanczos algorithm with truncated singular value regularization was run with no reorthogonalization of the Lanczos vectors.

This problem is only mildly ill-conditioned. For $n=25$ the calculated condition number (the ratio of the largest and smallest nonzero singular values) was $O\left(10^{2}\right)$, and the maximum error after one singular value was dropped was less than $3 \times 10^{-3}$. For $n=49$, the estimated condition number based on $k=40$ Lanczos steps was also of this order. In this case, best results for the solution (giving maximum error less than $10^{-3}$ ) were obtained by dropping no singular values.

Figure 2 shows the results for $n=97$ with $k=25$ and 40 . The condition number for $k=40$ was $O\left(10^{3}\right)$. One singular value was dropped for $k=40$, none for $k=25$.

Example 2. This is a time series problem drawn from the field of acoustic emission. The kernel, plotted in Fig. 3, is the theoretical displacement response of a certain horizontal elastic plate [14] to a vertical step function force term applied at a point on one of the faces. The response is measured directly below the force, on the opposite face of the plate. The kernel was sampled at $n=512$ points, and convolved numerically with a discretization of the function shown in Fig. 7. The resulting function, truncated to eight bits, was used as g. Elements in $g$ ranged from 0 to 53, and the residual norm $\|K f-g\|_{\infty}$ was $10^{-1}$. Figure 4 shows the results of using forward substitution on the linear system $K f=g$ to solve the time series deconvolution problem. This demonstrates the need for regularization of the solution. 


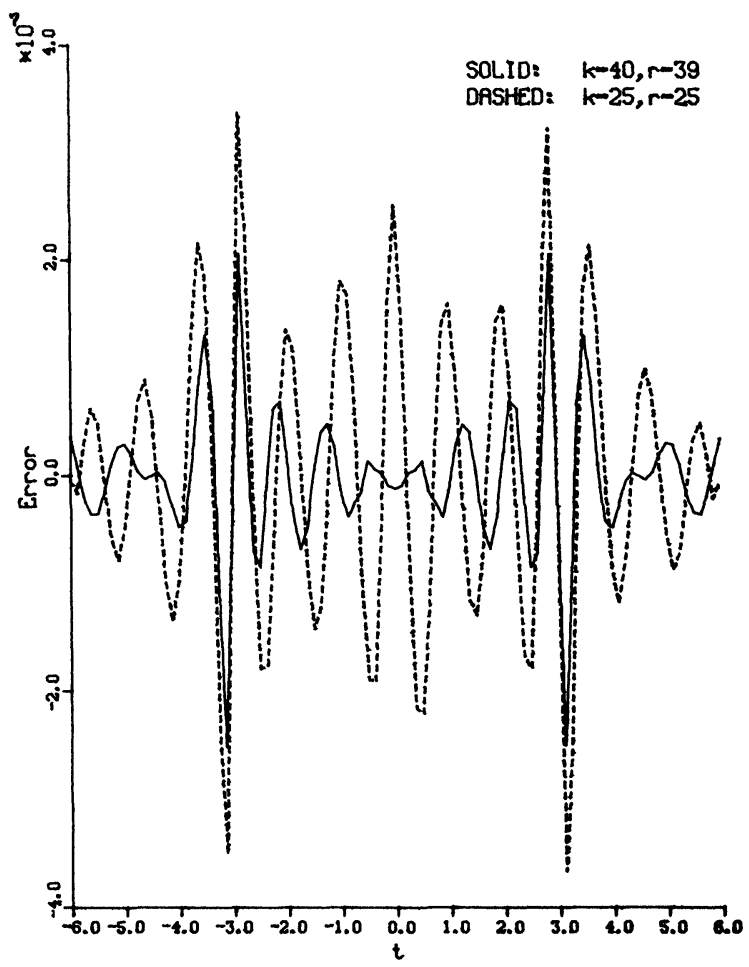

FIG. 2. Example 1, error in computed solution, $n=97$.

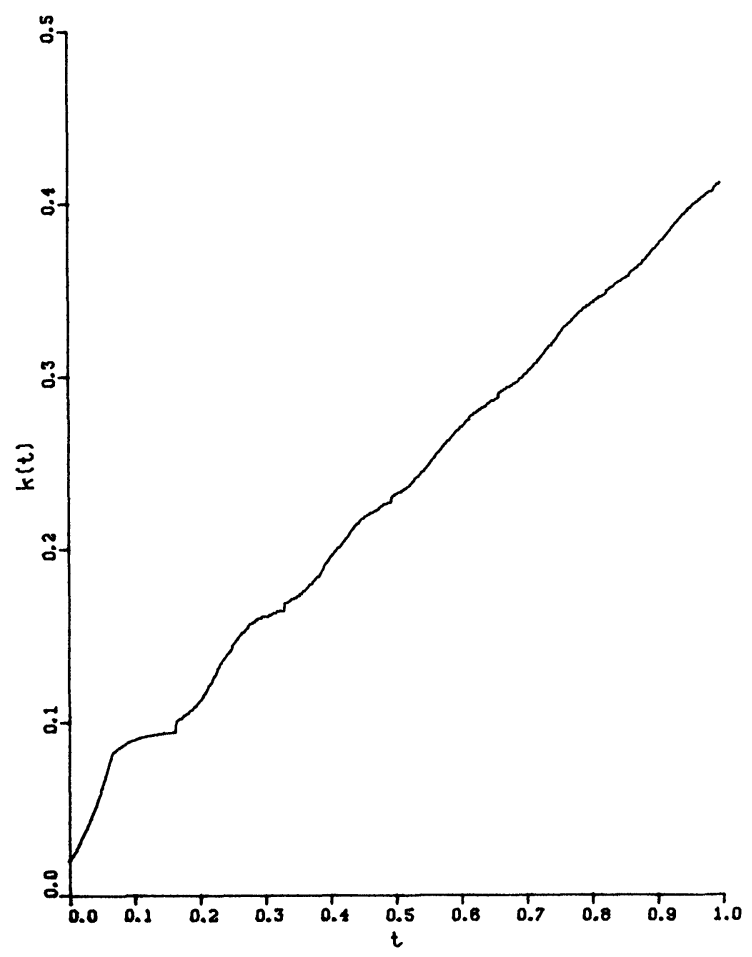

FIG. 3. Example 2, kernel function. 


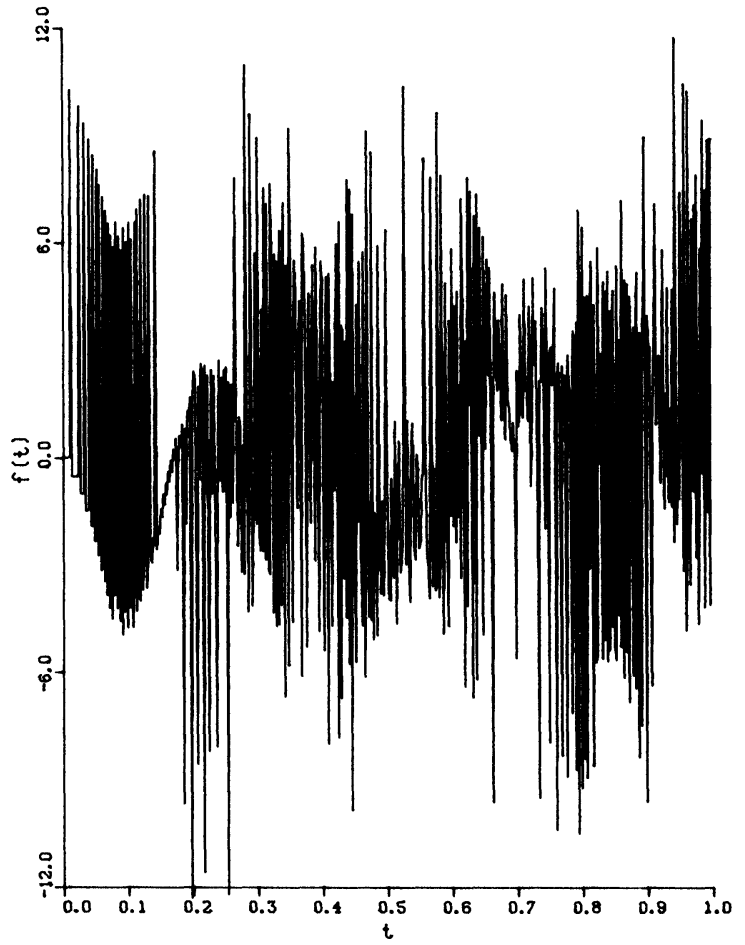

FIG. 4. Example 2, forward substitution solution.

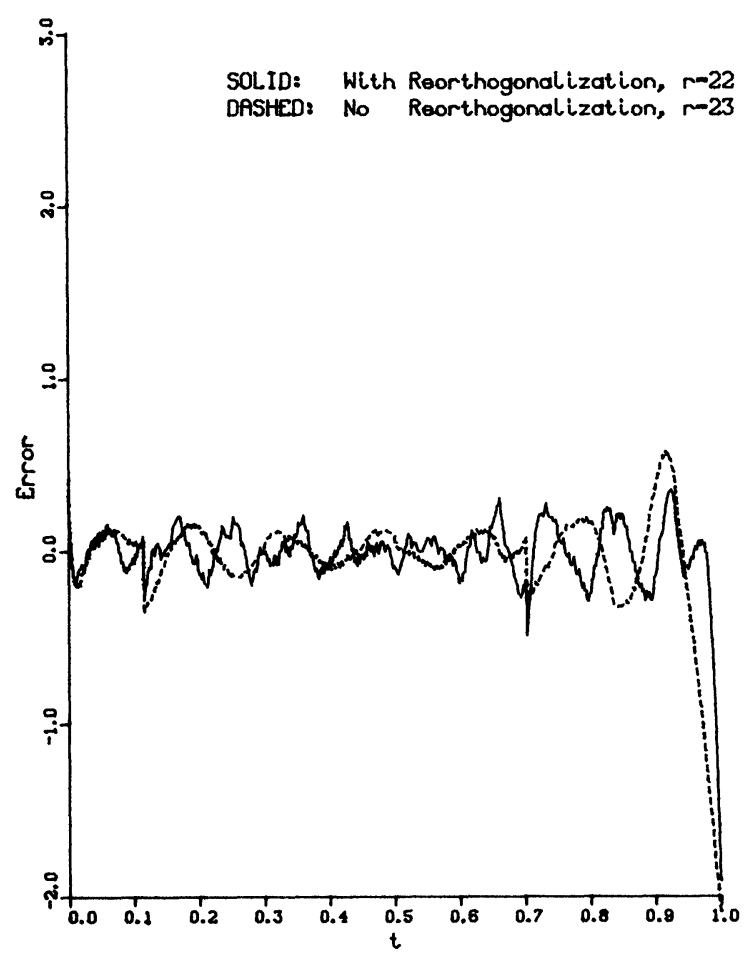

FIG. 5. Example 2, error in computed solution, $k=25$. 


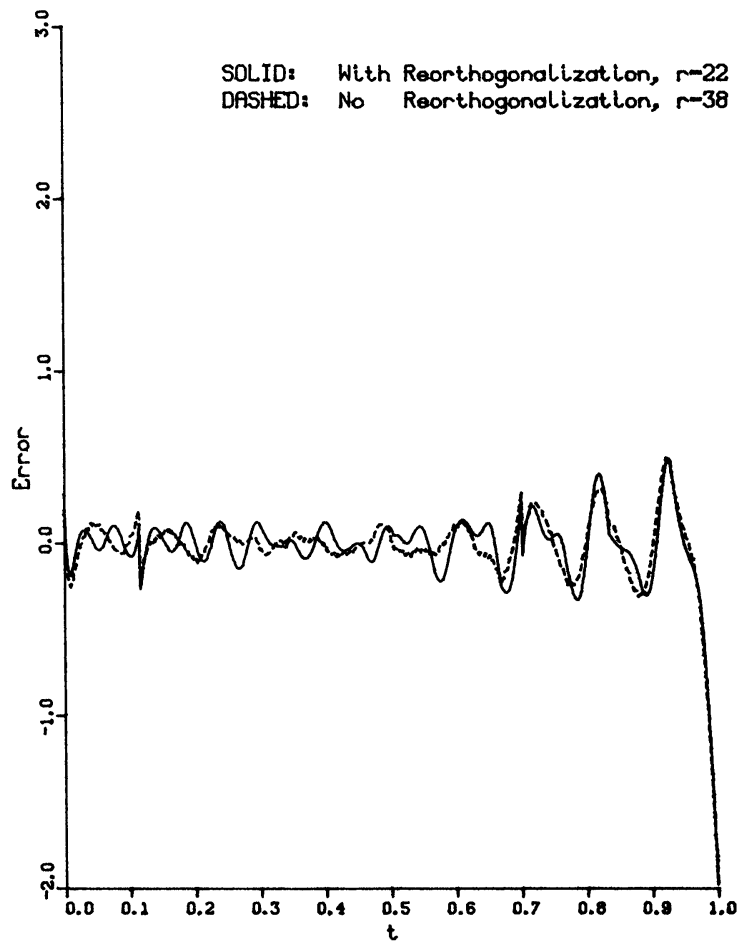

FIG. 6. Example 2, error in computed solution, $k=40$.

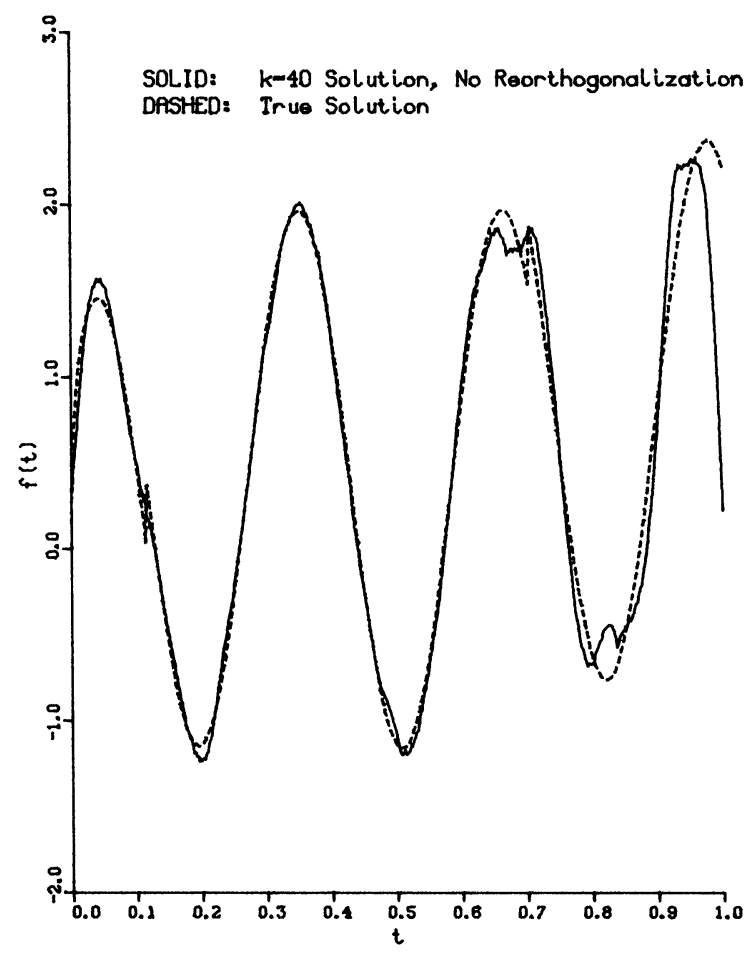

FIG. 7. Example 2, sample solution. 
The results in Fig. 5 were obtained using $k=25$ steps of the Lanczos algorithm with and without complete reorthogonalization. Three singular values were dropped in the calculation with reorthogonalization, two without. The estimated condition numbers were $O\left(10^{4}\right)$.

Analogous results for $k=40$ are shown in Fig. 6, dropping 18 singular values when reorthogonalization is performed, and 2 without reorthogonalization. Figure 7 is a comparison of the true and computed solutions without reorthogonalization. By this stage, significant roundoff error has entered into the nonreorthogonalized process, as evidenced by the appearance of spurious copies of several singular values. See [5], [15] for an explanation of this phenomenon. This meant that fewer small singular values appeared, but despite the complete loss of orthogonality of the Lanczos vectors, a good solution vector could still be reconstructed.

Acknowledgment. We are grateful to Martin R. Cordes for very helpful discussions, for expert assistance with mathematical software and for his careful reading of the manuscript. Helpful comments were made by G. W. Stewart and Jane Cullum. Some of this work was carried out under a joint program in acoustic emission studies between the National Bureau of Standards and the Electric Power Research Institute.

\section{REFERENCES}

[1] O. Axelsson, Solution of linear systems of equations: iterative methods, in Sparse Matrix Techniques, Copenhagen 1976, V. A. Barker, ed., Springer-Verlag, New York, 1977, pp. 1-51.

[2] Åke BJÖRCK, A bidiagonalization algorithm for solving ill-posed systems of equations, Rep. LiTHMAT-80-33, Dept. Mathematics, Linköping University, Linköping, Sweden, 1980.

[3] ÅKE BJÖRCK AND LARS EldÉn, Methods in numerical algebra for ill-posed problems, Rep. LiTHMAT-R-33-1979, Dept. Mathematics, Linköping University, Linköping, Sweden, 1979.

[4] P. Concus, G. H. Golub AND D. P. O'LeARY, A generalized conjugate gradient method for the numerical solution of elliptic partial differential equations, in Sparse Matrix Computations, J. R. Bunch and D. J. Rose, eds., Academic Press, New York, 1976, pp. 309-322.

[5] J. Cullum AND R. A. WilloughBy, The Lanczos tridiagonalization and the conjugate gradient algorithms with local, $\varepsilon$-orthogonality of the Lanczos vectors, Rep. RC 7152, IBM T. J. Watson Research Center, Math. Sciences Dept.,Yorktown Heights, NY, 1978.

[6] LARS ELDÉN, Algorithms for the regularization of ill-conditioned least squares problems, BIT, 17 (1977), pp. 134-145.

[7] — A program for interactive regularization part I: Numerical algorithms, Rep. LiTH-MAT-R-7925, Dept. Mathematics, Linköping University, Linköping, Sweden, 1979.

[8] W. GANDER, On the linear least squares problem with a quadratic constraint, Tech. Rep. STAN-CS-78697, Computer Science Dept., Stanford University, Stanford, CA, 1978.

[9] G. H. Golub, M. HEATH AND G. WAHBA, Generalized cross-validation as a method for choosing a good ridge parameter, Technometrics, 21 (1979), pp. 215-223.

[10] G. H. GOLUB AND W. KAHAN, Calculating the singular values and pseudo-inverse of a matrix, SIAM J. Numer. Anal., 2 (1965), pp. 205-224.

[11] G. H. Golub, F. T. Luk AND M. L. Overton, A block Lanczos method to compute the singular values and corresponding singular vectors of a matrix, Tech. Rep. STAN-CS-77-635, Computer Science Dept., Stanford University, Stanford, CA, 1977.

[12] F. G. GUSTAVSON AND D. Y. Y. YUN, Fast algorithms for rational hermite approximation and solution of Toeplitz systems, IEEE Trans. Circuits and Systems, CAS-26 (1979), pp. 750-754.

[13] R. W. Hamming, Digital Filters, Prentice-Hall, Englewood Cliffs, NJ, 1977.

[14] N. N. HSU AND S. C. HARDY, Experiments in acoustic emission waveform analysis for characterization of $A E$ sources, sensors, and structures, in Elastic Waves and Non-destructive Testing of Materials, Y. H. Pao, ed., AMD 29, American Society of Mechanical Engineering, 1978.

[15] W. KAHAN AND B. N. PARLETT, How far should you go with the Lanczos process? in Sparse Matrix Computations, J. R. Bunch and D. J. Rose, eds., Academic Press, New York, 1976, pp. 131-144.

[16] C. LANCzos, An iteration method for the solution of the eigenvalue problem of linear differential and integral operators, J. Res. Nat. Bur. Standards, 45 (1950), pp. 255-281. 
[17] C. LANCzOS, Solution of systems of linear equations by minimized iterations, J. Res. Nat. Bur. Standards, 49 (1952), pp. 33-53.

[18] S. LJUNG, A fast algorithm to solve Fredholm integral equations of the first kind with stationary kernels, Rep. LiTH-ISY-I-0265, Dept. Electrical Engin. Linköping University, Linköping, Sweden, 1979.

[19] C. B. Moler AND G. W. STEWART, An efficient matrix factorization for digital image processing, (manuscript) Computer Science Department, University of Maryland, College Park, MD, 1978.

[20] C. C. PAIGE, Bidiagonalization of matrices and solution of linear equations, SIAM J. Numer. Anal., 11 (1974), pp. 197-208.

[21] - Computational variants of the Lanczos method for the eigenproblem, J. Inst. Maths. Applics., 10 (1972), pp. 373-381.

[22] - Practical use of the symmetric Lanczos process with reorthogonalization, BIT, 10 (1970), pp. 183-195.

[23] J. PAlmer, Conjugate direction methods and parallel computing, Ph.D. Thesis, Stanford University, Stanford, CA, 1974.

[24] B. N. PARLETT AND D. S. SCOTT, The Lanczos algorithm with selective reorthogonalization, Math. Comp., 33 (1979), pp. 217-238.

[25] D. L. Phillips, A technique for the numerical solution of certain integral equations of the first kind, J. Assoc. Comput. Mach. 9 (1962), pp. 84-97.

[26] M. A. SAUNDERS, Sparse least squares by conjugate gradients: a comparison of preconditioning methods, Rep. SOL 79-5, Stanford University, Systems Optimization Laboratory, Stanford, CA, 1979.

[27] G. W. STEWART, Error and perturbation bounds for subspaces associated with certain eigenvalue problems, SIAM Rev., 15 (1973), pp. 727-764.

[28] Grace WAHBA, Numerical and statistical methods for mildly, moderately, and severely ill-posed problems with noisy data, Tech. Rep. 595, Dept. of Statistics, University of Wisconsin at Madison, 1980.

[29] J. H. Wilkinson, The Algebraic Eigenvalue Problem, Clarendon Press, Oxford, 1965. 